\title{
Assembly of colloidal aggregates by electrohydrodynamic flow: Kinetic experiments and scaling analysis
}

\author{
W. D. Ristenpart, I. A. Aksay, and D. A. Saville* \\ Department of Chemical Engineering, Princeton University, Princeton, New Jersey 08544, USA
}

(Received 7 October 2003; published 27 February 2004)

\begin{abstract}
Electric fields generate transverse flows near electrodes that sweep colloidal particles into densely packed assemblies. We interpret this behavior in terms of electrohydrodynamic motion stemming from distortions of the field by the particles that alter the body force distribution in the electrode charge polarization layer. A scaling analysis shows how the action of the applied electric field generates fluid motion that carries particles toward one another. The resulting fluid velocity is proportional to the square of the applied field and decreases inversely with frequency. Experimental measurements of the particle aggregation rate accord with the electrohydrodynamic theory over a wide range of voltages and frequencies.
\end{abstract}

DOI: 10.1103/PhysRevE.69.021405

PACS number(s): 82.70.Dd, 47.65.+a

\section{INTRODUCTION}

In its traditional form, the electrophoretic deposition of colloidal particles is analogous to sedimentation [1]. Since the electric field is a surrogate for gravity, (positively) charged particles move in the direction of the applied field. However, extensive experimentation during the last two decades has shown that particle motion transverse to the applied electric field occurs near electrodes under a variety of conditions, as long as particles do not adhere to the surface [2-7]. As a consequence of the transverse motion, aggregation takes place and the particles form densely packed planar clusters. Behavior of this sort is unexpected since the electrostatic interactions between identical particles-both Coulombic and induced dipole-are repulsive. Trau et al. $[4,7]$ attributed the transverse motion to an electrohydrodynamic (EHD) mechanism wherein fluid flow carries particles towards one another. Particles near an electrode alter the local electric field and the action of these perturbations on the electrode polarization charge layer yields fluid motion. They showed experimentally that if the particles are monodisperse and spherical, the transverse movement can create two- and three-dimensional colloidal crystals in both dc and low frequency $(\omega<10 \mathrm{kHz})$ ac fields. Bohmer's observations of ordering in dc fields [5] and the work of Yeh et al. [6] in dc and ac fields confirm the pervasive nature of the phenomenon. Aggregation of this sort has been observed with a variety of particles including nanoparticles [8,9] and biological cells $[10,11]$. Nonspherical particles aggregate via EHD flow as well $[9,10]$, though the aggregates may not always be crystalline. Given its ubiquitous nature, the phenomenon should find diverse applications $[12,13]$.

Although it is generally agreed that electric-field induced fluid flow plays a central role in moving the particles together, the detailed mechanisms remain somewhat unclear. Trau et al.'s [4] EHD model is applicable in both ac and dc fields and consistent with experimental studies on homog-

*Electronic address: dsaville@ princeton.edu enous electrodes as well as observations of particle motion toward ultraviolet-illuminated (high current) regions of semiconductor electrodes [14]. Their model has also proved useful in understanding the formation of crystals with large interparticle separations [15-17], as well as superlattices in binary suspensions [18]. However, the theory was not elaborated to provide quantitative predictions of particle aggregation behavior.

To describe aggregation in dc fields, Solomentsev et al. [19] proposed an alternative mechanism based on electroosmosis wherein flow due to the action of the steady field on the equilibrium diffuse layers around the particles draws them together. Since electroosmosis of this sort reverses in concert with the applied field and disappears at frequencies above a few hundred $\mathrm{Hz}$, the model does not pertain to aggregation in ac fields. Sides $[20,21]$ extended Trau et al.'s EHD model to investigate flow structure and the attendant particle movement in the vicinity of an electrode with a strong faradaic current, but concentration polarization at the electrode was ignored.

In this work, we focus on aggregation of spherical particles in fields oscillating at frequencies of several hundred $\mathrm{Hz}$ or higher. First, scaling expressions are derived to describe the EHD flow engendered by field inhomogeneities near the polarization layer. The free charge density is modeled for a perfectly polarizable electrode with an alternating potential, neglecting the presence of particles. Then the point dipole approximation is used to describe perturbations in the field near the electrode due to the particles, taking account of mobile charge in the particle double layer. This produces lateral body forces in the polarization layer. Combining these ingredients yields a simple scaling expression for the EHD velocity that brings particles together. According to the analysis, the aggregation rate scales with the square of the field strength and inversely with frequency. We anticipate similar behaviors as to field strength and frequency with more complex particle shapes.

To test the predictions of the scaling model, experimental techniques were developed to track the movements of a multitude of particles using video microscopy and image analysis. Then the disappearance of singlets (nonaggregated par- 


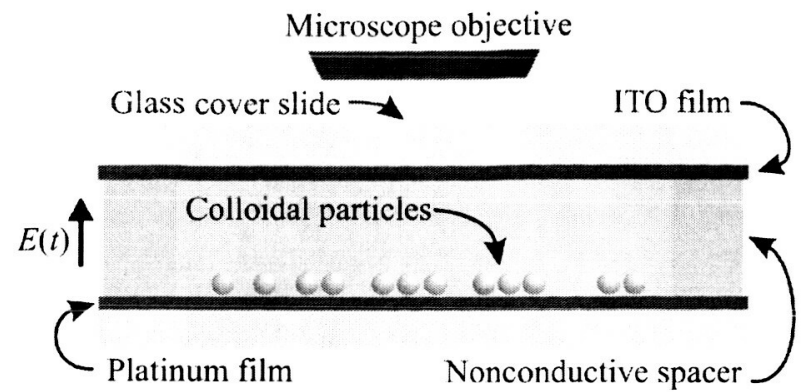

FIG. 1. A schematic diagram of the experimental apparatus (not to scale). A thin non-conductive spacer forms a cavity between two electrodes. The upper electrode is transparent-glass with a thin indium-tin-oxide layer-to facilitate optical microscopy. The lower silicon electrode is coated with a reflective layer of platinum.

ticles) was measured as a function of time over a wide range of field strengths and frequencies. The data were interpreted in terms of a second-order aggregation rate constant to establish the effects of the main electric field parameters (applied potential and frequency). Finally, comparison of the EHD model with experimental results shows that the scaling theory provides an accurate picture.

\section{SCALING THEORY}

To begin, consider the pure electrolyte between parallel electrodes (Fig. 1). When an electric potential is applied, counterions are attracted toward the electrodes and coions repelled. If electrochemical reactions are absent, the electrodes are "perfectly polarizable" and no current crosses a liquid-solid interface. As the applied potential varies in time, ion concentrations change to form and reform electrical double layers. Thus, charge on an electrode is balanced by charge in the diffuse layer. In the oscillating field, the fluid remains motionless and the pressure between the electrodes adjusts itself to maintain equilibrium. Solving the hydrostatic equation where pressure is balanced by electrical stresses with

$$
\mathbf{0}=-\nabla P+\rho^{(f)} \mathbf{E}
$$

and

$$
\rho^{(f)}=-\varepsilon \varepsilon_{o} \nabla^{2} \phi \quad \text { and } \quad \mathbf{E}=-\nabla \phi
$$

shows that the pressure and the potential gradients are related as

$$
p(z, t)=-\frac{1}{2} \varepsilon \varepsilon_{o}(\nabla \phi)^{2}+c .
$$

Here, the applied potential oscillates at a frequency $\omega / 2 \pi$, i.e., $\phi=\phi_{o}(z) \cos (\omega t) ; \varepsilon$ is the fluid dielectric constant, and $\varepsilon_{o}$ is the permittivity of free space. Since we are dealing with incompressible fluids, the constant of integration $c$ can be taken as the pressure in the absence of the field, $p_{o}$. Equation (3) represents electrohydrostatic equilibrium and, since $2 \cos ^{2}(\omega t)=1+\cos (2 \omega t)$, the laterally homogeneous osmotic pressure arising from the action of the field on electric charge consists of a steady component plus a fluctuation os- cillating at twice the imposed frequency. It follows as a corollary that lateral disturbances to the uniform charge distribution cannot be balanced solely by pressure changes and flow ensues to provide the necessary viscous stress.

The aggregation processes studied here arise from flow caused by the presence of small particles near an electrode but largely outside the charge layer. The particles perturb the otherwise uniform field, and the action of these perturbations on the charge in the polarization layer creates flow. It is stressed that the flow mechanism differs from classical electroosmosis in both the origin and dynamics of the charge distribution. In the classical model, the mobile equilibrium charge in the fluid balances charge that is chemically bound to solid-liquid interfaces. Here, the charge in the fluid fluctuates in response to the imposed, time-dependent potential. Because the induced charge is proportional to the applied field, electrical stresses scale on the square of the field and the response is justifiably designated as an electrohydrodynamic flow [22].

The relation between the tangential electrical stresses and velocity is revealed by inspecting the HelmholtzSmoluchowski relation for steady electroosmosis in the equilibrium diffuse layer along a rigid, charged interface [23]. For the frequency range of interest, the charge layer thickness is characterized by the Debye scale, $\kappa^{-1}$ [cf. Eq. (10)], and within the charge layer electrical stresses are balanced by viscous shear. For a 1-1 electrolyte,

$$
\kappa^{-1}=\sqrt{\varepsilon \varepsilon_{o} k_{B} T / 2 n_{\infty} e^{2}} .
$$

The symbols stand for the bulk electrolyte concentration, $n_{\infty}$; the charge on a proton, $e$; Boltzmann's constant, $k_{B}$; and the absolute temperature, $T$. In a $1-\mathrm{mM}$ salt solution, $\kappa^{-1} \sim 10 \mathrm{~nm}$. Outside the Debye layer, the fluid velocity asymptotes to the Helmholtz-Smoluchowski value

$$
u^{*}=-\frac{\varepsilon \varepsilon_{o} \zeta}{\mu} E_{t}
$$

Here, $\zeta$ denotes the electrostatic potential at the electrode solid-fluid interface, $\mu$ is the fluid's shear viscosity, and $E_{t}$ is the tangential component of the applied electric field. For a surface with $\zeta<0$, the action of the field on the positively charged counterions produces fluid movement in the direction of the field. It is instructive to rewrite this expression as a balance between the electric and viscous stresses on the Debye scale, i.e.,

$$
\mu \frac{u^{*}}{\kappa^{-1}}=-\frac{\varepsilon \varepsilon_{o} \zeta}{\kappa^{-1}} E_{t}=q E_{t},
$$

with Gauss's law employed to relate the potential gradient and $q$, the total charge per unit area in the diffuse layer. According to Eq. (6), the induced velocity is proportional to the electrical stress per unit area, $q E_{t}$. This proportionality will be used shortly to describe the velocity induced by perturbations in the polarization charge.

Further progress towards analytical expressions is facilitated by linearization of the various conservation laws. First, 
we calculate the charge in the polarization layer and then the EHD velocity. Hollingsworth and Saville [24] analyzed a linearized model of a dilute electrolyte between parallel plates with an applied potential difference, $\Delta \phi$, oscillating at a frequency of $\omega / 2 \pi$. The potential is given by (the real part of)

$$
\phi(z, t)=\frac{\Delta \phi}{2}\left\{1-\frac{\sinh (\gamma \hat{z})(\operatorname{csch} \gamma)-i \hat{z} \gamma \nu^{2} \operatorname{coth} \gamma}{1-i \gamma \nu^{2} \operatorname{coth} \gamma}\right\} e^{-i \omega t}
$$

where the various terms are defined as follows:

$$
\begin{gathered}
\Delta \phi=\left.\phi\right|_{z=-h}-\left.\phi\right|_{z=h}, \\
\gamma^{2}=(\kappa h)^{2}-i \frac{\omega h^{2}}{D}, \\
\nu^{2}=\frac{\omega}{\kappa^{2} D} .
\end{gathered}
$$

Here, the electrode plate separation is $2 h$, with $\hat{z}=z / h$, and $D$ represents the ion diffusivity.

For systems with $\kappa a \gg 1$, particles of radius $a$ maintain an average particle-to-surface gap that depends on a balance of several forces, including electrostatic repulsion, van der Waals attraction, hydrodynamic effects, and gravity. Our observations show that particle movement in the electric field consists of Brownian motion superimposed on a directed translation. The rapidity of the Brownian fluctuations suggests that the particle-electrode gap is substantial. This is consistent with total internal reflection microscopy measurements by Fagan et al. [25], who found that the mean separation distance is several Debye lengths, even in the presence of a strong oscillatory field. Outside the polarization layer, particles experience a uniform field directed perpendicular to the electrodes and from Eq. (7) this is

$$
\begin{aligned}
\mathbf{E}_{\infty} & =-\left.\nabla \phi\right|_{z=0}=\frac{\Delta \phi}{2 h}\left\{\frac{\gamma \operatorname{csch} \gamma-i \gamma \nu^{2} \operatorname{coth} \gamma}{1-i \gamma \nu^{2} \operatorname{coth} \gamma}\right\} e^{-i \omega t} \mathbf{k} \\
& \sim \frac{\Delta \phi}{2 h} e^{-i \omega t} \mathbf{k} .
\end{aligned}
$$

Here, $\mathbf{k}$ denotes a unit vector in the $z$ direction, cf. Fig. 2. Inasmuch as $\gamma^{2} \sim(\kappa h)^{2} \gg 1$ for $\omega / 2 \pi \ll 10^{7} \mathrm{~Hz}$, the field strength is mostly in-phase with the applied potential.

To implement the scaling suggested by Eq. (6), we need the charge in the electrode polarization layer. In the frequency range of our experiments,

$$
\kappa^{2} D \sim 10^{7} \mathrm{~Hz} \gg \omega \gg \kappa D / h \sim 400 \mathrm{~Hz},
$$

the free charge calculated from Eq. (7) is (a)

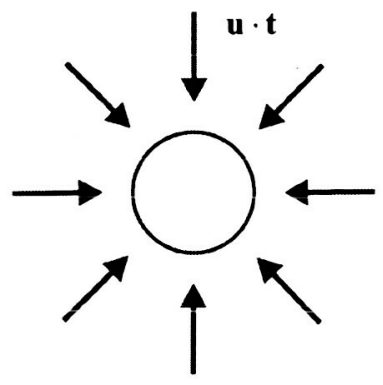

(b)

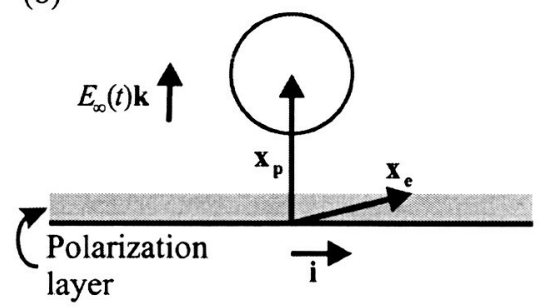

FIG. 2. Definition sketch depicting a spherical particle near an electrode. (a) The plan view depicting flow towards the test particle under conditions where the inequality $\left[C_{o}^{\prime}+\left(D \kappa^{2} / \omega\right) C_{o}^{\prime \prime}\right]<0$ is satisfied. (b) An elevation view showing a particle located at $x_{p}$ outside the polarization layer; $\mathbf{x}_{\mathbf{e}}$ denotes a point in the polarization layer near the electrode, with $\mathbf{x}_{\mathbf{e}} \cdot \mathbf{k} \approx 0$.

$$
\begin{aligned}
q & =\left.\varepsilon \varepsilon_{o} \frac{\partial \phi}{\partial z}\right|_{z=-h} \\
& =\frac{\varepsilon \varepsilon_{o} \Delta \phi}{2 h}\left\{\frac{\gamma \operatorname{coth} \gamma-i \gamma \nu^{2} \operatorname{coth} \gamma}{1-i \gamma \nu^{2} \operatorname{coth} \gamma}\right\} e^{-i \omega t} \\
& \sim \frac{\varepsilon \varepsilon_{o} \Delta \phi}{2 h}\left\{1+i \nu^{-2}\right\} e^{-i \omega t} .
\end{aligned}
$$

Of course the effects of suspended particles are also important but these are difficult to capture in detail without analyzing the electrohydrodynamics of the region between an electrode and nearby particles.

Next, to obtain the lateral component of the electric field due to a colloidal particle, we portray the particle's influence in terms of a "point-dipole approximation." The electric potential around a sphere in the uniform field well outside the diffuse layer (Fig. 2) is readily shown to be the real part of

$$
\phi=-a E_{\infty}\left[\left(\mathbf{x}-\mathbf{x}_{\mathbf{p}}\right) \cdot \mathbf{k}-C_{o} \frac{\left(\mathbf{x}-\mathbf{x}_{\mathbf{p}}\right) \cdot \mathbf{k}}{r^{3}}\right] e^{-i \omega t}
$$

Here, $E_{\infty}=\Delta \phi / 2 h$ is the incident field strength, $\mathbf{x}$ and $\mathbf{x}_{\mathbf{p}}$ are vectors (scaled with the particle radius) for position and the location of the center of the particle, $r$ is the (scaled) distance from the particle center, and $C_{o}$ is the (dimensionless, complex) "dipole coefficient." Recall that for a dielectric particle in a dielectric medium, the dipole coefficient is a real quantity, given by the Clausius-Mossotti formula, viz., $\left(\varepsilon_{p}\right.$ $-\varepsilon) /\left(\varepsilon_{p}+2 \varepsilon\right)$ [26]. Here, we are interested in charged particles suspended in an electrolyte where $C_{o}$ is a complex 


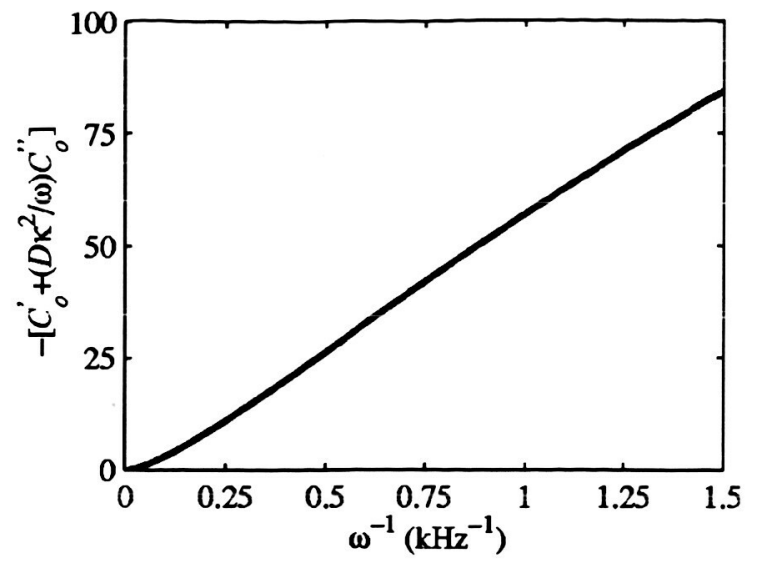

FIG. 3. The dipole coefficient proportionality from Eq. (14) as a function of inverse frequency for a particle with a $-120-\mathrm{mV}$ surface potential and $1-\mu \mathrm{m}$ radius in $1-\mathrm{mM} \mathrm{KCl}$.

function of frequency and the properties of the particle ( $\zeta$-potential or surface charge and radius) and electrolyte (ionic strength, ion valences and mobilities, and dielectric constant). Accordingly, the dipole coefficient will be computed using the standard electrokinetic model [23].

At $\mathbf{x}_{\mathrm{e}}$, a point close to the electrode (near $z=-h$ ), the $x$ component of the electric field around the dipole is the real part of

$$
\begin{aligned}
\mathbf{E} \cdot \mathbf{i} & =3 E_{\infty} C_{o} \frac{\left[\left(\mathbf{x}_{\mathbf{e}}-\mathbf{x}_{\mathbf{p}}\right) \cdot \mathbf{k}\right] \mathbf{x}_{\mathbf{e}} \cdot \mathbf{i}}{r^{5}} e^{-i \omega t} \\
& \approx-3 E_{\infty} C_{o} \frac{\left(\mathbf{x}_{\mathbf{p}} \cdot \mathbf{k}\right)\left(\mathbf{x}_{\mathbf{e}} \cdot \mathbf{i}\right)}{r^{5}} e^{-i \omega t}
\end{aligned}
$$

To establish the scaling of the EHD body force and its direction, we proceed as follows. From Eqs. (10) and (12) and the properties of the trigonometric functions, the electric stress in the $x$ direction at $\mathbf{x}_{\mathbf{e}}$ has a steady component proportional to

$$
q E_{t} \sim 3 \varepsilon \varepsilon_{o} \frac{(\Delta \phi)^{2}}{4 h^{2}}\left\{C_{o}^{\prime}+\frac{D \kappa^{2}}{\omega} C_{o}^{\prime \prime}\right\} \frac{\left(\mathbf{x}_{\mathbf{p}} \cdot \mathbf{k}\right)\left(\mathbf{x}_{\mathbf{e}} \cdot \mathbf{i}\right)}{r^{5}}
$$

along with an oscillation at twice the imposed frequency. It follows that the tangential velocity due to $\mathrm{EHD}$ forces scales as

$$
\mathbf{u} \cdot \mathbf{t} \sim \frac{3 \varepsilon \varepsilon_{o}}{\mu \kappa} \frac{(\Delta \phi)^{2}}{4 h^{2}}\left\{C_{o}^{\prime}+\frac{D \kappa^{2}}{\omega} C_{o}^{\prime \prime}\right\}
$$

According to Eq. (14), the direction of the steady EHD motion is established by the real and imaginary parts of $C_{o}\left(e \zeta / k_{B} T, \kappa a, \omega a^{2} / D, \ldots\right)$; flow will be towards the "test particle" if $\left[C_{o}^{\prime}+\left(D \kappa^{2} / \omega\right) C_{o}^{\prime \prime}\right]<0$. Since the dipole coefficient is a weak function of frequency over this range, the velocity scales approximately as $\omega^{-1}$. Figure 3 shows the outcome of a numerical calculation using the standard electrokinetic model [27]. Since the proximity of the electrode and the suspended particles will also influence matters, detailed numerical calculations will be useful to provide a complete quantitative picture of effects of ionic strength, particle size, etc. Nevertheless, the scale analysis furnishes an accurate picture of the effects of field strength and frequency.

Equation (14) also provides a means to interpret the rate constant, $k_{E}$, of a second order aggregation process in electrohydrodynamic terms (cf. Sec. III). If particles are carried towards one another by flow, then the flux towards a test particle across a circle of radius $r$ is proportional to $2 \pi r u(r) n(r)$, where $n(r)$ represents the singlet particle concentration. Hence, the rate of aggregation per unit area will be second order in the number of particles per unit area with a rate constant whose magnitude behaves as

$$
k_{E} \approx 2 \pi a \frac{3 \varepsilon \varepsilon_{o}}{\mu \kappa} \frac{(\Delta \phi)^{2}}{4 h^{2}}\left|C_{o}^{\prime}+\frac{D \kappa^{2}}{\omega} C_{o}^{\prime \prime}\right|
$$

Thus, the rate of clustering is proportional to the square of the applied field $(\Delta \phi / 2 h)^{2}$, and inversely proportional to the frequency $\omega / 2 \pi$. Field strength and frequency are the main external control variables. As the experiments described in Sec. III demonstrate, this is the observed behavior.

\section{EXPERIMENTS}

\section{Apparatus and materials}

The experimental cell consisted of two parallel electrodes separated by a 500- $\mu \mathrm{m}$ poly-dimethylsiloxane (PDMS) spacer (Fig. 1). The upper (grounded) electrode was glass with a thin coating of indium tin oxide, an optically transparent semiconductor $(R \sim 4-8 \Omega$, Delta Technologies); the lower silicon electrode was coated with a 150 -nm-thick layer of platinum (Radiant Technologies). To clean the system, the electrodes and spacer were sonicated for $10 \mathrm{~min}$ in RBS-35 detergent (Pierce Chemicals), thoroughly rinsed, sonicated again for $10 \mathrm{~min}$ in deionized water, and then dried with filtered nitrogen gas. Two batches of $2.7-\mu \mathrm{m}$ monodisperse polystyrene particles (Interfacial Dynamics Corp.) with different surface charge densities were used. The particles were washed and diluted with the appropriate electrolyte solution to volume fractions of approximately 0.01 .

To begin an experiment, a suspension was placed in the cell and particles allowed to settle to form a dilute layer near the bottom electrode. Measurements of Brownian diffusion indicated that the particle-electrode separation was several hundred nanometers. Evidently the particles are kept out of contact with the electrode by a combination of electrostatic and steric effects. Oscillatory fields were then applied with a Tektronix function generator (PFG5505) and measured with a Tektronix digital oscilloscope (TDS2012). After acquiring a sequence of digital images at different stages in the clustering process, the voltage was turned off. Although Brownian motion breaks up particle clusters after aggregation it is a slow process; here a high frequency, high amplitude ac field was employed to expedite matters. Particle positions were observed with a reflection microscope (Mitutoyo) and recorded using a CCD camera and a digital framegrabber. 


\section{Image analysis and kinetic interpretation}

Particle location was the main observable and, at low magnifications, 500-1000 particles could be imaged simultaneously. We chose to analyze the behavior of a very large number of particles instead of focusing on a few binary events. Brownian motion often brings particles into close proximity and then separates them; concentrating on the behavior of a few particles can be misleading.

At the outset, the majority of the particles were isolated (i.e., not in apparent contact with other particles) and undergoing Brownian motion. Upon application of the field, "singlets" moved together or toward larger aggregates to form clusters (Fig. 4). The observed motion was approximately two-dimensional and the rate of singlet disappearance served as a measure of the rate of aggregation induced by the electric field. Since our focus is on the initial rate of particle aggregation, the crystallites are relatively small; it is clear from previous work that they will assemble into larger twoor three-dimensional crystals [4].

To obtain the rate of singlet disappearance, images were recorded at one frame per second. Then standard image analysis techniques, including a thresholding step that separated individual particles (and higher order aggregates) from the background, were applied to each image. This yielded a list of clusters of various sizes and their locations. Although the use of low magnification complicates an accurate assessment of the number of particles in a given cluster, singlets have a relatively narrow distribution of "detected" sizes. A cluster that subtended $p$ pixels $\left(p_{\min }<p<p_{\max }\right)$ was identified as a singlet; clusters with $p>p_{\max }$ were identified as higher order clusters. Inspection of a number of singlets in representative images indicated that the method is very accurate, with the majority of errors introduced at the edge of the image. Therefore, particles adjacent to the image edges were excluded from the analysis.

Experiments at different field strengths indicate that the rate of singlet depletion is strongly affected by the characteristics of the electric field. To quantify the aggregation process, the rate of singlet disappearance can be expressed as

$$
\frac{d n_{1}}{d t}=-2 k_{11} n_{1} n_{1}-\sum_{j=1}^{N} k_{1 j} n_{1} n_{j}
$$

where $n_{j}$ represents the concentration (per unit area) of clusters containing $j$ particles, and $k_{1 j}$ is a rate constant (with units of area/time) governing the rate at which singlets aggregate with clusters of size $j$. The factor of 2 accounts for the loss of two singlets each time a doublet forms; cluster breakup is neglected. To further simplify matters, we focused on the initial rate where particle disappearance was dominated by singlet-singlet events since higher order clusters were few in number. Under these conditions Eq. (16) simplifies to

$$
\frac{d n_{1}}{d t}=-k_{E} n_{1}^{2}
$$

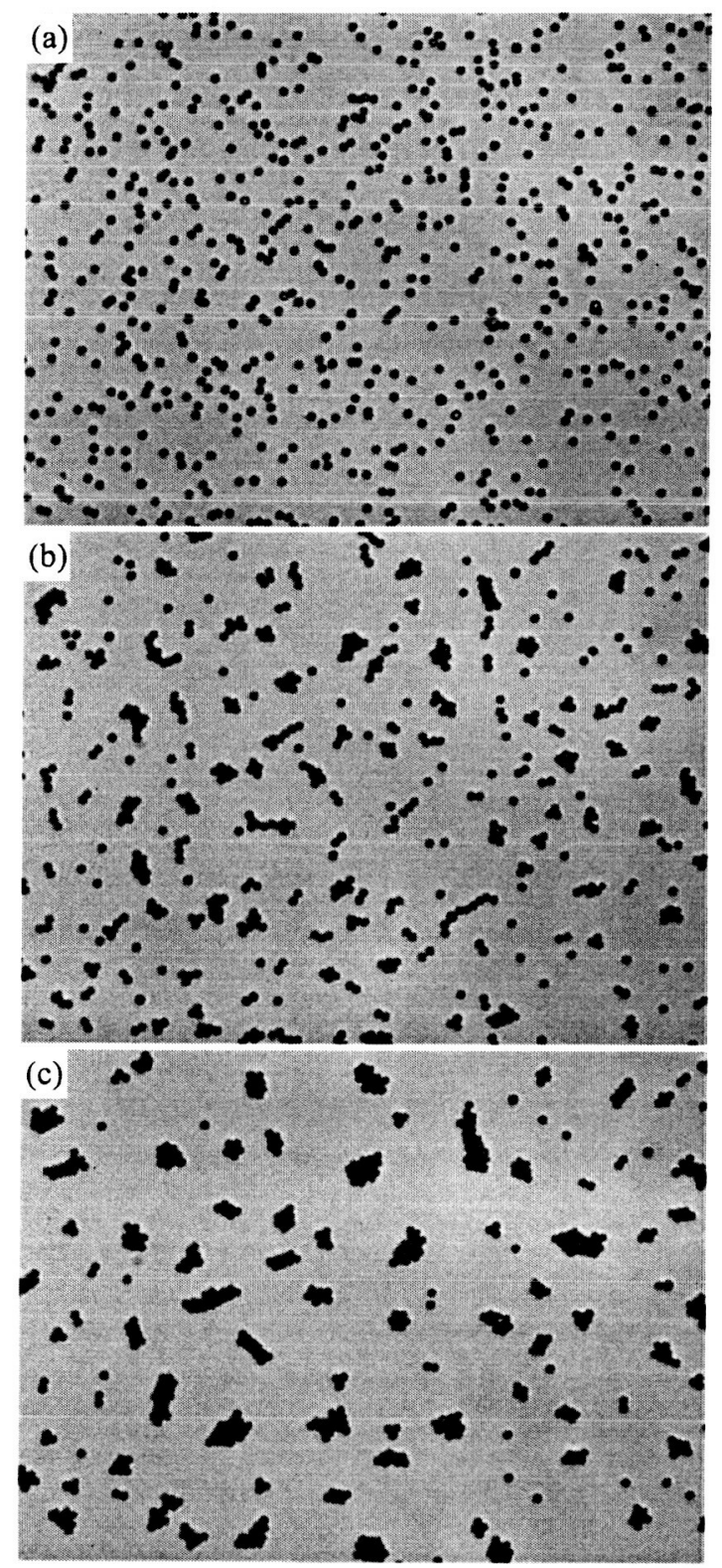

FIG. 4. Lateral aggregation in response to an electric field. Particles are $2.7-\mu \mathrm{m}$ polystyrene in $1.0-\mathrm{mM} \mathrm{KCl}$. The applied field is $500 \mathrm{~Hz}$ and $6 \mathrm{~V}$, oriented out of the page. (a) Initially there are 407 isolated particles ("singlets") dispersed throughout the field of view, with a few additional particles in momentary contact due to Brownian motion. (b) After $10 \mathrm{~s}$, the majority of particles have aggregated into clusters, with 87 singlets remaining. (c) After $30 \mathrm{~s}$, only 19 singlets remain.

$$
\frac{n_{1}^{o}}{n_{1}}=1+k_{E} n_{1}^{o} t
$$

The factor of 2 has been incorporated into the rate constant, $k_{E}$, and $n_{1}^{o}$ is the initial particle concentration. According to Eq. (18), the inverse singlet concentration increases linearly with time, with slope equal to $k_{E} n_{1}^{o}$. 


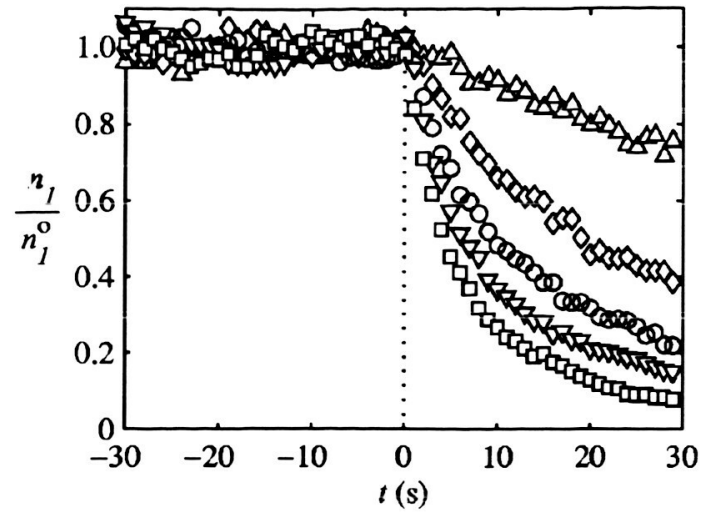

FIG. 5. Singlet concentrations (normalized on the initial concentration) as a function of time for different applied voltages at a frequency of $1 \mathrm{kHz}$. Squares, $6 \mathrm{~V}$; downward triangles, $5 \mathrm{~V}$; circles, $4 \mathrm{~V}$; diamonds, $3 \mathrm{~V}$; upward triangles, $2 \mathrm{~V}$. Upon application of the field, the singlet concentration decreases as aggregation ensues. Particles are $2.7-\mu \mathrm{m}$ polystyrene in $1.0-\mathrm{mM} \mathrm{KCl}$. The initial singlet concentration for each voltage is approximately one particle per $70 \mu \mathrm{m}^{2}\left(\sim 14000\right.$ particles $\left./ \mathrm{mm}^{2}\right)$.

\section{Aggregation kinetics}

By carrying out the analysis procedure on a sequence of images collected during an experiment, the number of singlets in a fixed viewing area was determined as a function of time. Representative data are shown in Fig. 5. To ensure that singlet-singlet events predominated, data analysis terminated when $20 \%$ of the singlets remained or $30 \mathrm{~s}$ after the field was applied, depending on which occurred first. As the data show, before application of the field, $n_{1}$ remains relatively constant, with small fluctuations as particles momentarily encounter one another due to Brownian motion. Upon application of the field, $n_{1}$ decreases rapidly with a rate dependent on the applied potential. Once the field is removed, the number of singlets increases as the aggregates disperse by Brownian motion (data not shown). Rescaling the data as $n_{1}^{o} / n_{1}$ (Fig. 6)

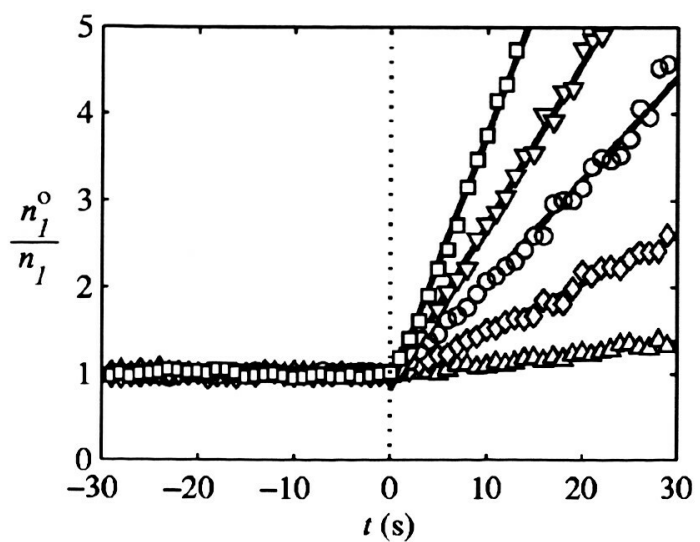

FIG. 6. Scaled (inverse) singlet concentration data from Fig. 5. Symbols are the same as in Fig. 5. Solid lines are linear fits to the data; the slopes of the lines yield the aggregation rate constants (see the text).

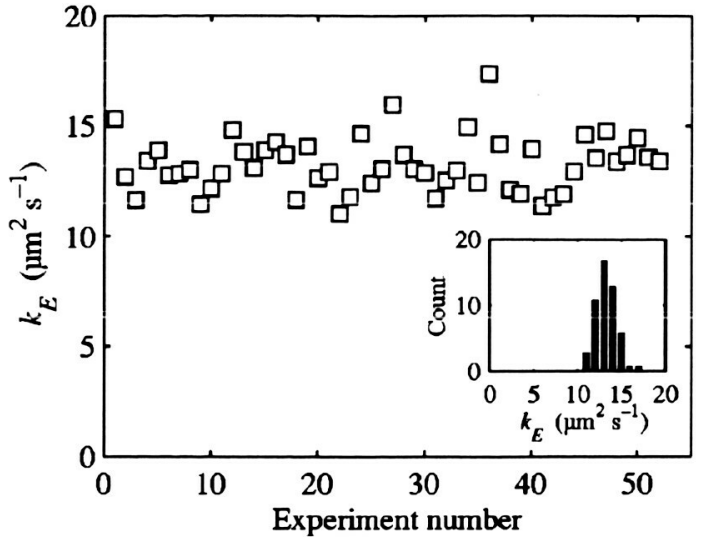

FIG. 7. Aggregation rate constants obtained from a series of 52 sequential experiments, each at $500 \mathrm{~Hz}$ and $4 \mathrm{~V}$. A histogram (inset) shows the number of experiments with a given rate constant.

shows that the inverse concentration increases linearly with time, consistent with Eq. (18).

To test reproducibility, a sequence of 52 experiments was carried out with a $500-\mathrm{Hz}, 4-\mathrm{V}$ applied potential. As Fig. 7 indicates, the rate constants are quite reproducible. A histogram (Fig. 7, inset) suggests a quasi-Gaussian distribution with a standard deviation of (approximately) $10 \%$ of the mean. Preliminary experiments indicated that one cause for the scatter is variation in the initial particle configuration. If, on average, particles are much closer to one another (i.e., higher concentrations) at the onset of the electric field, then the apparent rate of aggregation will be correspondingly larger due to higher order encounters. To minimize this effect, the particles were moved into a widely separated configuration prior to each experiment using a $10-\mathrm{kHz}, 10-\mathrm{V}$ applied field. This provided a reproducible random initial condition for each experiment. However, it was difficult to precisely control the initial singlet concentration, which varied between experiments due to random aggregation and deaggregation via Brownian motion. This partially accounts for the scatter in the measured rate constants, since contributions from higher order clusters are neglected in the analysis.

To confirm that the particles were randomly positioned at the start of an experiment, the degree of disorder was quantified using a well-defined "translational order measure," $T$; $T=0$ for perfect disorder and $T=1$ for perfect order [28]. In the set of 52 experiments, the average value of $T$ was $0.024 \pm 0.014$, indicating that the ac field procedure was effective in arranging the particles into disordered initial configurations.

\section{COMPARISON OF THEORY AND EXPERIMENTS}

To probe the validity of the scaling expression set out in Eq. (15), the aggregation rate was measured as a function of the field strength and frequency. Rate constants (extracted from the data illustrated in Fig. 6) are plotted against the square of the applied voltage amplitude for frequencies of 500 and $1000 \mathrm{~Hz}$ (Fig. 8). Ten experiments were performed at each amplitude and frequency. The aggregation rate con- 


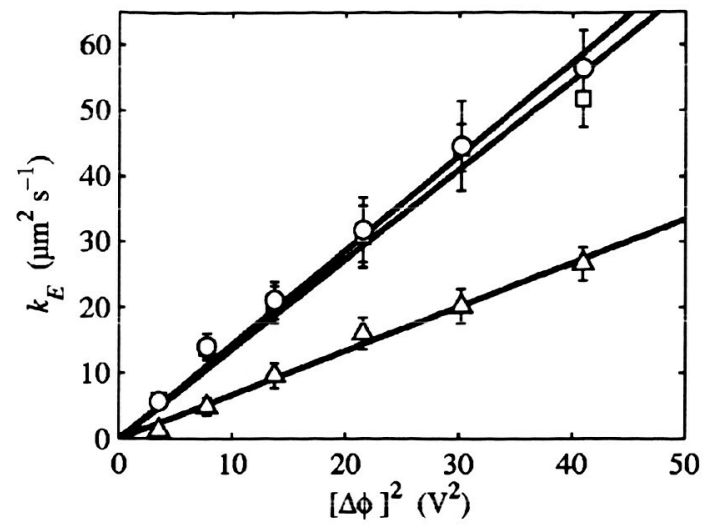

FIG. 8. Aggregation rate constant-applied potential relation. Particles are $2.7-\mu \mathrm{m}$ polystyrene in $1.0-\mathrm{mM} \mathrm{KCl}$. Each point is the mean of ten separate experiments; error bars represent one standard deviation. Two sets of experiments were done at $500 \mathrm{~Hz}$ with particles of same size but different electrophoretic mobilities: circles, $M=-8.6 \mu \mathrm{m} \mathrm{cm} / \mathrm{V} \mathrm{s}$; squares, $M=-9.5 \mu \mathrm{m} \mathrm{cm} / \mathrm{V} \mathrm{s}$. Another set of experiments was carried out at $1000 \mathrm{~Hz}$ (triangles). Solid lines were derived by linear regression.

stant increases linearly with $(\Delta \phi)^{2}$, in agreement with the scale analysis, and this dependence extends over an order of magnitude at both frequencies. The $(\Delta \phi)^{2}$ dependence was replicated with a different particle, albeit with a similar $\zeta$ potential (Fig. 8). It is reassuring to note that the numerical magnitude of the measured rate constant $k_{E} \approx 10^{-11} \mathrm{~m}^{2} / \mathrm{s}$ (Fig. 8), is consistent with that estimated using Eq. (15).

Another series of experiments was employed to investigate the effect of frequency (Fig. 9). For polystyrene particles in $1.0-\mathrm{mM} \mathrm{KCl}$, the rate constant changed as $1 / \omega$ for frequencies larger than $1000 \mathrm{~Hz}$, in agreement with Eq. (15). The solid lines are correlations for frequencies greater than 1 $\mathrm{kHz}$, chosen because it is an order of magnitude larger than the minimum frequency $\kappa D / H \sim 100 \mathrm{~Hz}$ for which the scaling analysis is valid. The inverse frequency dependence found here is similar to that identified by Kim et al. [29], who found a $\omega^{-1.2}$ dependence between 30 and $500 \mathrm{~Hz}$ for $10-\mu \mathrm{m}$ polystyrene spheres in $0.1-\mathrm{mM} \mathrm{NaHCO} 3$. A key difference is that we observe a maximum aggregation rate at $\sim 500 \mathrm{~Hz}$, with the rate constant decreasing as $\omega \rightarrow 0$ (data not shown). Since the inverse frequency dependence is only valid for frequencies much greater than $\sim 100 \mathrm{~Hz}$, it is not surprising that slightly different behavior is observed at lower frequencies. One explanation for diminished aggregation rates at low frequencies may be that significant vertical electrophoretic motion is present; such motion was clearly observable as particles moved slightly in and out of the focal plane. Electrochemical reactions may also play a role at lower frequencies $[20,25]$. At higher frequencies, induced dipole-dipole repulsion tends to inhibit aggregation [15-18], and presumably affects the maximum frequency at which particles aggregate (i.e., the $k_{E}=0$ intercepts in Fig. 9).

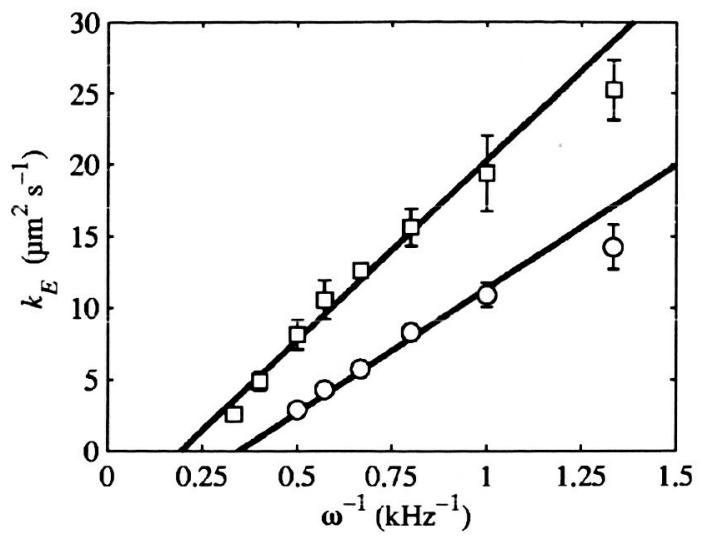

FIG. 9. Aggregation rate constant-frequency relation for two applied potentials. Symbols: circles, $\Delta \phi=4 \mathrm{~V}$; squares, $\Delta \phi$ $=6 \mathrm{~V}$. Each data point is an average from ten experiments; error bars are one standard deviation. Solid lines are linear fits for frequencies of $1 \mathrm{kHz}$ and larger (inverse frequencies of $1 \mathrm{kHz}^{-1}$ or smaller).

Nonetheless, over the range of frequencies where electrophoretic motion is negligible and the free charge is given by Eq. (10), the rate of aggregation decays inversely with frequency.

\section{CONCLUSIONS}

Scaling expressions for the EHD fluid velocity engendered by the dipole field of a polarized particle were derived and it was shown that the direction of flow depends on the sign of the dipole coefficient. According to the analysis, the velocity is proportional to the square of the applied potential and inversely proportional to the frequency. To test these scaling predictions, the behavior of large numbers of particles was tracked and the aggregation kinetics quantified in terms of a second-order rate constant. The experimental results are all consistent with the scaling analysis.

Although the analysis presented here focused on oscillatory fields and monodisperse suspensions, the approach is applicable in other situations. For example, EHD flow occurs in steady fields, although analysis is complicated by several additional effects (e.g., electrochemical reactions and electroosmotic flow [19]). In addition, the scale analysis can be extended to binary or ternary suspensions [18] although more detailed models will be required to describe such systems.

\section{ACKNOWLEDGMENTS}

This work was supported by NASA OBPR, the NASA University Research, Engineering, and Technology Institute on BioInspired Materials (BIMat) under Award No. NCC-102037, NSF/DMR-0213706, and DARPA. Partial support for W.D.R. was provided by NASA GSRP. 
[1] H. Hamaker and E.J.W. Verwey, Trans. Faraday Soc. 36, 180 (1940).

[2] P. Richetti, J. Prost, and P. Barois, J. Phys. (France) Lett. 45, 1137 (1984).

[3] P. Richetti, J. Prost, and N. A. Clark, in Physics of Complex and Supermolecular Fluids, edited by S. Safran and N. A. Clark (Wiley, New York, 1987), p. 387.

[4] M. Trau, D.A. Saville, and I.A. Aksay, Science 272, 706 (1996).

[5] M. Bohmer, Langmuir 12, 5747 (1996).

[6] S.R. Yeh, M. Seul, and B.I. Shraiman, Nature (London) 386, 57 (1997).

[7] M. Trau, D.A. Saville, and I.A. Aksay, Langmuir 13, 6375 (1997).

[8] M. Giersig and P. Mulvaney, J. Phys. Chem. 97, 6334 (1993).

[9] T. Teranishi, M. Hosoe, T. Tanaka, and M. Miyake, J. Phys. Chem. B 103, 3818 (1999).

[10] A.T. Poortinga, R. Bos, and H.J. Busscher, Biotechnol. Bioeng. 67, 117 (2000).

[11] V. Brisson and R.D. Tilton, Biotechnol. Bioeng. 77, 290 (2002).

[12] O.D. Velev, T.A. Jede, R.F. Lobo, and A.M. Lenhoff, Nature (London) 389, 447 (1997).

[13] G.M. Whitesides and B. Grzybowski, Science 295, 2418 (2002).

[14] R.C. Hayward, D.A. Saville, and I.A. Aksay, Nature (London) 404, 56 (2000).
[15] T.Y. Gong and D.W.M. Marr, Langmuir 17, 2301 (2001).

[16] T.Y. Gong, D.T. Wu, and D.W.M. Marr, Langmuir 18, 10064 (2002).

[17] F. Nadal, F. Argoul, P. Hanusse, B. Pouligny, and A. Ajdari, Phys. Rev. E 65, 061409 (2002).

[18] W.D. Ristenpart, I.A. Aksay, and D.A. Saville, Phys. Rev. Lett. 90, 128303 (2003).

[19] Y. Solomentsev, M. Bohmer, and J.L. Anderson, Langmuir 13, 6058 (1997).

[20] P.J. Sides, Langmuir 17, 5791 (2001).

[21] P.J. Sides, Langmuir 19, 2745 (2003).

[22] D.A. Saville, Annu. Rev. Fluid Mech. 29, 27 (1997).

[23] W. B. Russel, D. A. Saville, and W. R. Schowalter, Colloidal Dispersions (Cambridge University Press, Cambridge, UK, 1991).

[24] A.D. Hollingsworth and D.A. Saville, J. Colloid Interface Sci. 257, 65 (2003).

[25] J.A. Fagan, P.J. Sides, and P.C. Prieve, Langmuir 18, 7810 (2002).

[26] J. D. Jackson, Classical Electrodynamics, 2nd ed. (Wiley, New York, 1975).

[27] E.H.B. Delacey and L.R. White, J. Chem. Soc., Faraday Trans. II 77, 2007 (1981).

[28] S. Torquato, T.M. Truskett, and P.G. Debenedetti, Phys. Rev. Lett. 84, 2064 (2000).

[29] J. Kim, S.A. Guelcher, S. Garoff, and J.L. Anderson, Adv. Colloid Interface Sci. 96, 131 (2002). 